\title{
Interactive Visible Light Communications (iVLC): Supporting Wireless Communications and Smart Visual Interaction with a Common Lighting Infrastructure
}

\author{
Marcos Katz \\ Centre for Wireless \\ Communications \\ University of Oulu \\ Oulu, Finland \\ marcos.katz@oulu.fi
}

\author{
Iqrar Ahmed \\ Centre for Wireless \\ Communications \\ University of Oulu \\ Oulu, Finland \\ iqrar.ahmed@oulu.fi
}

\author{
Jari Laru \\ Learning and Educational \\ Technologies \\ University of Oulu \\ Oulu, Finland \\ jari.laru@oulu.fi
}

\author{
Juha Häkkinen \\ Circuits and Systems Group \\ University of Oulu \\ Oulu, Finland \\ juha.hakkinen@oulu.fi
}

\begin{abstract}
The concept of interactive visible light communications (iVLC) is introduced and discussed in this paper. iVLC exploits a matrix of solid-state light luminaries in multiple ways, namely providing lighting and wireless communications as well as supporting visual signaling and interaction. Every individual element of the light matrix can be exploited in multiple domains, i.e., space, time, color, intensity and data. As a result, the lighting infrastructure works a flexible platform that can be exploited in wide range of applications and use cases. The paper discusses attractive applications of iVLC, particularly in the school of the future, health-care and office spaces. Multiple classroom tasks can be supported by iVLC, including orchestration of activities, feedback between students and teachers, dynamic grouping, etc.
\end{abstract}

Keywords - wireless communications, visual interactions, visual signaling, human-light interaction, RGB LED, LED matrix.

\section{INTRODUCTION}

The advent of solid-state lighting started a new era in the field of illumination. White light emitting diodes (LEDs) have rapidly displaced conventional lighting sources such as filament-, plasma- or gas-based lamps in most practical environments. White LEDs' unique advantages include high energy-efficiency, mechanical robustness, durability and high flexibility in terms of control. Predictions estimate that LED penetration will reach about $85 \%$ by 2030 and $100 \%$ around 2035 [1]. Today, the greatest incentives to use LEDs for lighting purposes are clearly the significant saving in energy and maintenance costs that can be achieved. Solid-state lighting (SSL) offers new possibilities and advantages to the end-user. By using appropriate drivers and a control strategy, the qualitative characteristics of the light can be controlled. Thus, it is possible to produce illumination in well-defined areas, adapting flexibly light intensity, distribution patterns and colour to different needs as well as to the human circadian rhythm. This will improve not only the energy efficiency but also user satisfaction of the lighting system.

The fact that LEDs can be electronically controlled to produce a variable optical output is very important and it can be exploited in different ways. If the control of the light acts slowly on the light characteristics, we refer to smart-light applications, with changes in lightning typically taking place in time scales measured from minutes to hours. On the other extreme, LEDs can be very rapidly controlled (e.g., on-off switched, complex modulated) being the speed of change mostly limited by the internal capacity of the diode junctions. This is the approach used by visible light communication (VLC) to transmit information optically. The optical output of the LED can be modulated by the information bearing signal using different techniques, from simple on-off keying (OOK) to more advanced ones like orthogonal frequency division modulation (OFDM) and color-shift keying (CSK), for example. In general, illumination and wireless communications have been considered separately by different research communities, though some joint work is available, as discussed later. One can also control the LEDs over a relatively short period of time (e.g., seconds to minutes) to produce another type of information, namely visual signaling aiming to interact quickly with people. This visual information that can be created with an array of controllable LEDs can be used, among others, to inform, guide, orchestrate, warn, support social interaction, indicate states or situations, and motivate people in the nearby surroundings. Unlike radio, visible light can convey direct and intuitive visible messages that are easy for people to understand. The three areas of operation, VLC, visual interaction and smart lighting, define the concept of interactive visible light communications (iVLC), as illustrated 
by Fig. 1. In this paper, the considered concept will be presented and discussed, and some attractive applications will be introduced. Note that these operating modes can be supported by a common lighting infrastructure, as will be discussed later. Moreover, the three modes can be simultaneously used.

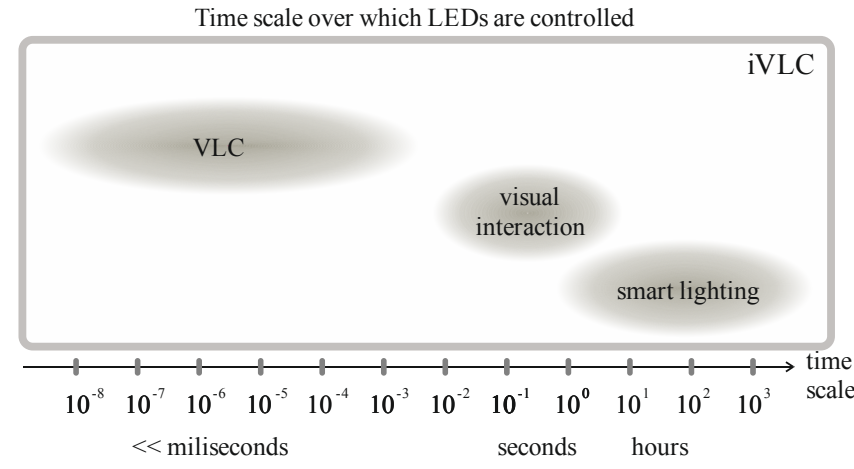

Fig. 1. The concept of iVLC comprises a) VLC, b) visual interaction and signalling, and c) smart lighting.

This paper is organized as follows. Section II overviews the joint use of VLC and lighting in general. Section III defines interactive VLC in more detail while Section IV focuses on the applications of the concept. Using iVLC in the classroom, hospitals and office spaces is discussed and exemplified. Section $\mathrm{V}$ discuss challenges of iVLC and Section VI concludes the paper.

\section{VLC AND ILLUMINATION}

Wireless and mobile connectivity is today a fundamental commodity used widely across the globe. The current worldwide penetration of mobile devices exceeds $100 \%$. The overwhelming majority of mobile and wireless access is based on the use of radio technology. This striking preponderance of radio is expected to continue in the future. However, the steady growth in the number of users as well as the explosive increase in the traffic have created a number of challenges for radio-based communications systems. Spectral limitation is one of the most remarkable challenges as the available spectrum is a welllimited natural resource that cannot easily serve a hyperconnected world eager for more and more information. The Internet of Things (IoT) paradigm, defining a world where trillions of objects could be wirelessly connected to internet, will further exacerbate the spectrum shortage. Many technical solutions have been proposed to fight against spectrum scarcity such as the use of multiple antennas (e.g., massive MIMO, beamforming techniques, etc), the use of coordinated transmission by exploiting several base stations, etc. These approaches are promising though their implementation lead often to solutions that are not necessarily cost-effective nor energy efficient. Moving towards less congested or unused frequency bands, particularly higher frequency bands, is a promising approach to get additional spectrum. This has occurred already in $5 \mathrm{G}$ and will be even more pronounced in $6 \mathrm{G}$, where millimetre-waves and tera-Hertz are considered. Moreover, the use of optical communications, VLC in particular, is a highly attractive option to the problem of spectrum shortage. In addition, VLC has unique advantages.
VLC is secure and private as the signal remains in the room, and it can support very high data rates. Possible interference and radio exposure problems, typical with radio systems, are irrelevant. Moreover, VLC is relatively simple to implement, it is inherently low power and inexpensive for low- and moderatebit rates. VLC is highlighted as an attractive technology be used in future 6G systems, complementing radio technologies [2].

In general, visible light communication has been studied extensively in the past decade. However, the design of VLC systems taking into account lighting considerations has not been deeply investigated. Authors in [3-4] study this case. While a single-transmitter-single-receiver data link using the VLC concept is relatively easy to implement, a multiple input multiple output (MIMO) VLC system is quite challenging as it requires intelligent handover and smart lighting. Cognitive illumination, discussed in [5] provides a way forward to support MIMO with VLC. Authors exploited the controllable nature of LEDs to design the cognitive optical cells for seamless communication fulfilling illumination requirements for multiple users. So far, white LEDs are mostly employed for illumination and nonlinearities can hamper the performance of white LEDs [6]. The effect of nonlinearities become highly relevant when LED are modulated. There is a tradeoff between signal strength and spectral efficiency while the LED performance can drop by $20 \%$ [6].

Certain illumination requirements are needed to be followed in office spaces. In fact, approximately 300 lux illumination is required to attain a comfortable and aesthetically pleasant experience to the human eye. In order to retain the LED's performance, DC-balanced modulated signals are used for driving LEDs [7]. Authors in [8] considered the illumination requirements when designing and implementing VLC systems. In general, the lighting system need to be designed to meet stringent requirements of communications (i.e., VLC) and lighting. Color requirements and minimum energy constraints are challenges in the aforementioned case.

Smart illumination [9-10] focus on achieving high energy efficiency while incorporating several advanced features in the system such as dimming, positioning, broadcasting and navigation. Authors in [11] consider intelligent road illumination for smart cities, where the dimming feature is used to adjust the illuminations of LED sources, aiming at minimizing energy expenditure. VLC is extensively researched nowadays due to ever-growing number of communication devices and the increasing popularity of advanced services leading to congestion of the radio frequency (RF) spectrum. The concept of building smart cities by exploiting the ubiquity of lights to ease the RF spectrum shortage, as presented in [12]. Apart from quality of service (QoS) in VLC, the illumination conditions are equally important in order to build intelligent ubiquitous cities. So far, achieving higher data rates has been the key goal of the VLC community, and different schemes and LED technologies have been developed. However, the paradigm of smart cities and VLC in daily life has shifted the focus towards meeting the stringent requirements of VLC and aesthetically pleasant light. 


\section{INTERACTIVE VISIBLE LIGHT COMMUNICATIONS}

We define here interactive visible light communications (iVLC) as an advanced platform used for a) conventional and smart lighting using solid-state lighting, b) providing wireless communications using the VLC principles and c) creating an intuitive and flexible visual interaction and signaling system, as depicted in Fig. 1. An RGB LED (Red Green Blue) can be electronically controlled to adjust its intensity, color (wavelength) as well as it can be modulated according to information to be transmitted. This can be extended to an array of RGB LEDs, where additionally one can exploit the temporal and spatial domains. Data transmission takes place by modulating the light output of the LED. This technique, known as VLC, can support hundreds of Mbps and beyond. Illumination and visual signaling requires a slow control of the luminaries, with slow fluctuations in the light output, typically measured from fraction of seconds to hours.

We assume that LEDs are arranged into a two-dimensional matrix, for instance, on the ceiling of a room, though the array can be distributed across the room in any possible threedimensional arrangement. LEDs then could represent light sources on the ceiling of a room. In practice lighting elements could also be on the floor, walls and integrated into furniture, appliances and objects, for instance. Figure 2 depicts the case the light sources are arranged as a matrix of $N$ times $M$ lighting elements. The light output $l$ of the generic $(x, y)$ element of the $N \times M$ light matrix $L$ can be controlled in the intensity ( $i$ ), color $(c)$, data $(d)$, space $(x, y)$ and time ( $t)$ domains, as seen in Fig. 2. The exploitation of light in these domains is the key characteristic of iVLC. Unlike radio, light is a powerful and highly intuitive physical phenomenon that can be easily detected and interpreted by human beings.

$(N \times M)$ light matrix $L$

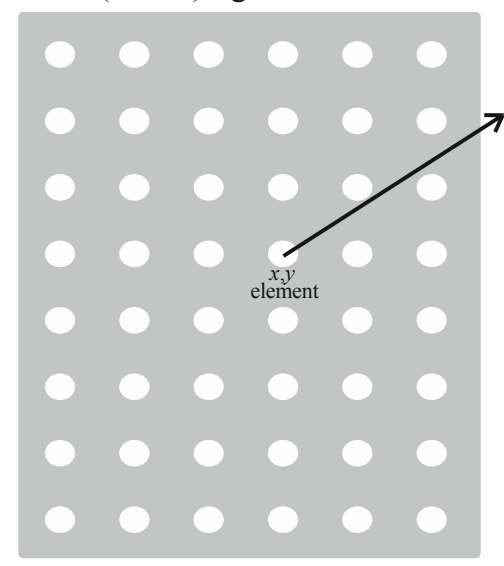

The light output of a given light element of $L$ can be written as

$l_{x, y}=f(c, i, d, x, y)$

$x=1,2, \ldots N$

$y=1,2, \ldots M$

$c$ : color

$i$ : intensity

d: data

1101010010

$x, y:$ space

$t$ : time the matrix inherently supports broadcasting of information. Multibeam transmission can also be used, aiming at achieving larger data throughput or better link reliability.

In order to exploit the interactional aspects of iVLC using the considered light matrix concept, one needs to resort to defining human-light-interaction (HLI), which, in the same spirit of human-computer interaction, can be defined as the design of lighting technologies and visual messaging concepts aimed to convey light-based information to human beings (i.e., users). In other words, HLI defines how certain target actions are mapped into light outputs. A target action is a desired activity or situation an actor wants to achieve. The action consists of an act lasting a given period of time, short or long. An action could be simple such as sending a given piece of (visual) information to a particular user in a room. A simple example would be keeping room illumination at a given intensity level and colour for a given period of time. Another action could be an elaborated succession of activities defining for instance certain orchestration. A target action could be for instance broadcast certain information to people, provide visual guiding, orchestrate a complex situation, create a particular mood, illuminate the room, display a graphic symbol or icon (still or moving), send data to a particular device or group of devices, etc. It is important to note that several target actions can be realized at the same time, for instance, while illuminating a given scenario, the system can also provide data coverage as well as produce visual signalling in the time-space-colourintensity domains.

Figure 3 illustrates how HLI can be practically implemented using a matrix of RGB LEDs. A number of visual actions or light output are defined in the time, space, colour, intensity and data domains. Each output corresponds to a given input or target action. The HLI mapping block in Fig. 3 maps a target action, e.g., work group orchestration in a classroom, into a series of meaningful light actions signalling users what to do or how to behave. Both, inputs and light outputs depend on the scenario or use case in particular. For a given scenario, all input/output pair need to be identified. Light outputs should be intuitive, unambiguous and motivating Creating a visual language of interaction would be the first step to develop any system. The meaning of colours, shapes, spatio-temporal light patterns and other visual signalling in each context should be well defined. One should consider also that the semantics of a visual language used as described here has a strong relationship with the cultural background of the users. A colour associated with warning may vary in different cultures, for instance. Research on anthropology of light and its effect on human light would shed more light on how to create the most effective human-light interaction methodologies. Development of HLI languages would support development of flexible programming tools.

Fig. 2. Light output of a generic element of a $\mathrm{N} x \mathrm{M}$ light matrix.

From the VLC standpoint, this matrix arrangement has multiple advantages. First, uniform coverage across the room can be achieved. The approach also supports spatial separation of users, as users in different locations can be served by beams generated by different LEDs, improving SNR and enhancing privacy in multi-user environments, among others. Certainly, 


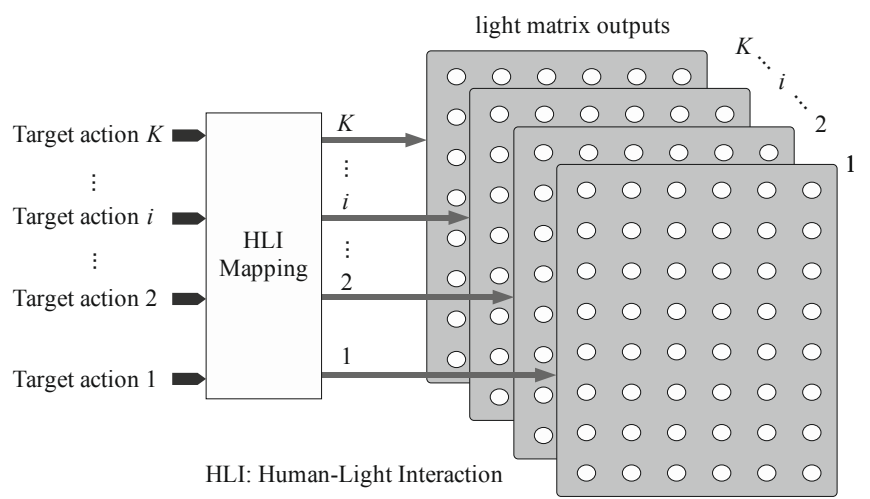

Fig. 3. Mapping target actions into light outputs.

Figure 4 shows examples of how target actions (inputs) can be mapped into outputs of a iVLC system. Of course, since the tempoporal domain cannot be displayed, these examples are just simplifications. In a) localized feedback is exemplified, showing negative (red) and positive (green) visual feedback to users located below the luminaries. These could be student in a class room. In b) localized data and feedback is shown. In case c) an example of grouping activity is shown, where each coloured spatial area indicates users belonging to a given group. In d), normal uniform (white-light) lighting is shown, while e) the colour has been tuned to create certain atmosphere prone to have an effect on the users, e.g., relaxation, enhancing attention, etc. Data broadcasting example across the service area is depicted in example f). In g) a smart-lighting case of energysaving mode is shown. According to the distributiuon of users, time of the day or other needs, the lighting pattern and intensity of the light matrix can be controlled. In h) the matrix is used as a large (low-definition) display, where icons, text and other information can be displayed. The color profile of the matrix can also be changed to create particular atmospheres or effects related to the activities being carried out in the service area. In j) a joint visual signalling (green arrow) and transmission of multi-user information is shown.
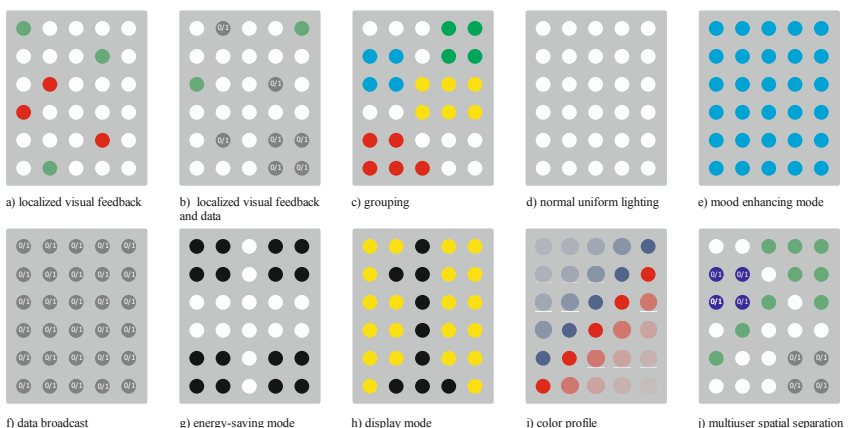

Fig. 4. Mapping target actions into light outputs.

\section{ApPLiCATIONS}

In this section, we consider applications for the iVLC concept. In principle, iVLC can be used in any scenario where wireless connectivity needs to be provided and there is a need to interact visually with users in the service area. Multiple scenarios have these requirements. Here we highlight three demanding environments where the concept of iVLC would be highly attractive, namely learning environments, health-care environments and office spaces/home environments.
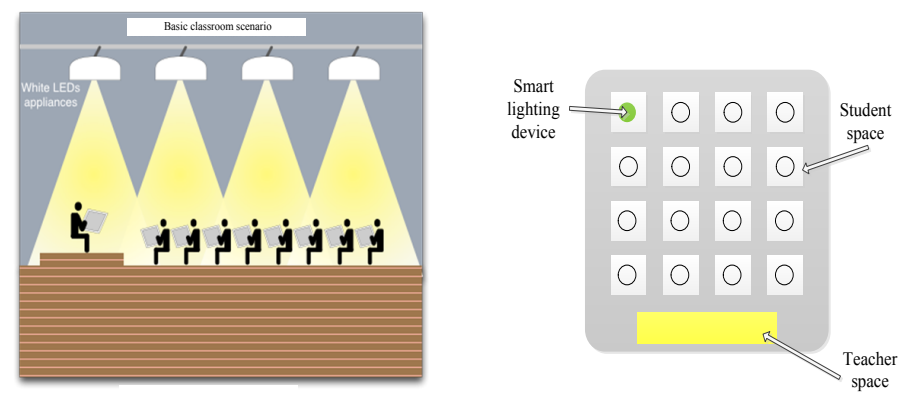

Fig. 5. Classroom using iVLC (left), lighting devices spatially aligned with students (right).
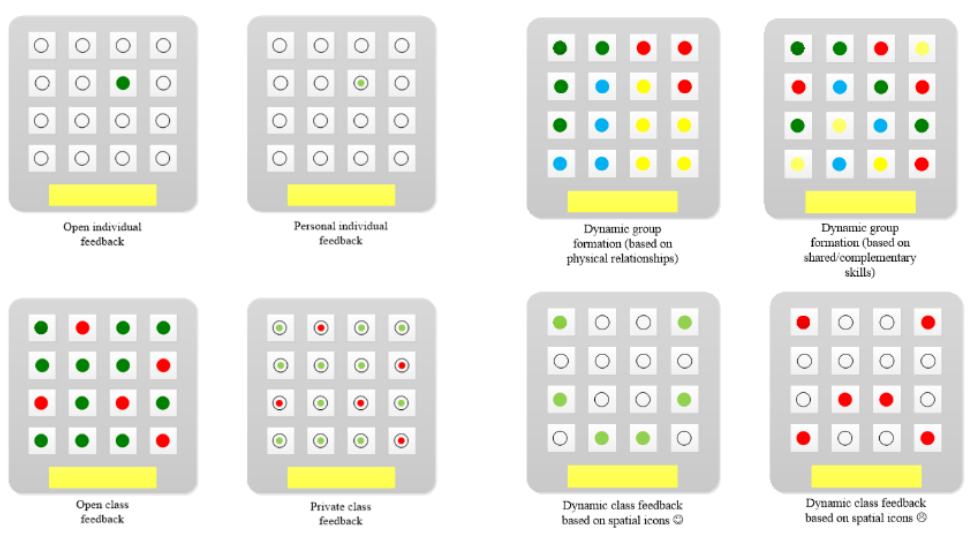

Fig. 6. Examples of the use of iVLC in a class-room.

\section{Learning Environments:}

By learning environment, it is understood any scenario where people interact with one or more teachers and with each other in order to learn. As such, a learning environment can be a school, university, training center, etc. A classroom is the typical place where learning take place. Figure 5 illustrates a classroom served by an iVLC system. One can assume there is one luminaire above each student defining a personal lighting space, though other arrangements are possible. The most typical situation occurs when all personal lighting spaces are covered by white light, in a way the room is well lit. On top of this, the HDI will create communication and visual signaling to manage situations in the classroom, such as orchestrating intuitively and in an encouraging manner teaching activities, providing information channels between students and the teacher to distribute learning material, real-time feedback (visual- and data-based), enhancing social interaction in the class (studentsgroups-teacher), etc.

Figure 6 depicts examples of interaction/signaling in a classroom. The two first examples show how individual feedback can be given, either visually or through VLC data. For 


\section{Before teaching \\ Design for learning \\ - Pedagogical scripts \\ - Technological agents \\ - Core contents (topics)}

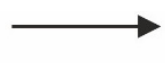

Learning phase

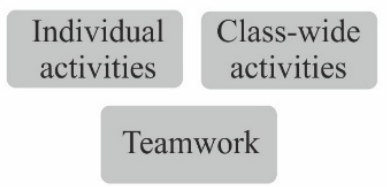

Evaluation phase

Interaction analysis

- Replay of activities (afterwards)

- Scaffolding, evaluation and reflection,

to increase awareness and enable regulation

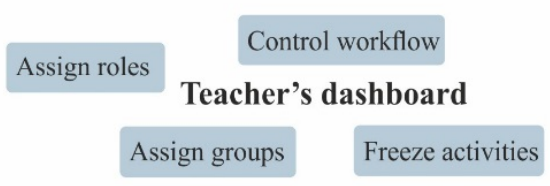

Fig. 7. Teacher-student interaction in a classroom exploiting iVLC.

instance, if the feedback to a student is positive, an instantaneous (green) light blink can be produced above the student. But a negative feedback needs not to be openly visible, and can be provided through data. Feedback can also be given to the whole classroom, similarly, using visible signals or data. Finally, Fig. 7 provides an example of a possible pedagogical script for the classroom, by which certain activities could be implemented and supported by the iVLC system [17]. On the left-hand side is the teacher's dashboard with a number of possible activities to be carried out during the leaning time. These include assigning groups, tasks and roles, controlling the workflow, freezing activities, and many others. Visual signaling and interaction using light as well as VLC data are the key elements to master the activities. On the right-hand side of Figure 7 one can see the students' corresponding activities, that can be seen on their devices (e.g., tablets) as well as light outputs from the lighting infrastructure. Student activities include working/rest time, visualization tasks, self-regulation, visual workflow and others. The school of the future will likely exploit advanced interactive and visualization systems integrated with their wireless and lighting infrastructure.

\section{Health-care Environments:}

In these operating scenarios interaction-rich activities take place in many forms. Moreover, both advanced smart-lighting and wireless communication technology will bring unique advantages. A hospital is perhaps the most typical and demanding environment to deploy and use iVLC technology. Different hospital spaces can be considered, patient wards, private rooms, intensive care units, operating theatres, medical imaging units, and others. Wireless connectivity is important in health-care environments as wireless-based systems not only provide information and other relevant data access but also, they bring freedom, easy-of-use, security and comfort to medical staff and patients as well. However, radio-based systems (e.g., cellular systems, Wi-Fi and other short-range communications) can cause interference to sensitive hospital equipment. Radio systems can be eavesdropped and jammed as well, so security and privacy are challenges for radio systems in hospitals. Light-based communications solve security, privacy and interference issues. Social interaction and visual signaling are also highly relevant in hospitals. Light provides connectivity as the patient can be remotely monitored. Realtime physiological information can be transferred from the patient to a health server using for instance infrared links (invisible to the human eye), while information to the patient such as equipment control data, patient-requested Internet data, and others are provided by the lighting infrastructure. In addition, if the patient requires attention (due to his/her own will or current heath condition) a suitable visual signaling above the bed is produced. Also, mood enhancing light can be set according to the needs of each patient. Upon the occurrence of an event or certain need (e.g., visit to nurse room, restroom, canteen or hospital evacuation), the lights will visually guide ward patients. The hospital of the future could also use iVLC systems widely across its spaces.

\section{Office/Working Spaces:}

Lighting plays a fundamental role in our working environments, and it is related to productivity and creativity. Light conditions at the workplace have their impact also on health, well-being and behavior of the personnel exposed to it. Changing the luminous conditions in office can affect office workers by changing the visual capability [13], visual comfort [14], and perception of the conditions [15]. Visual capability can influence task performance greatly [16]. Visual comfort has effect on mood that can influence feeling of health and wellbeing. Visual capability, visual comfort, and perception also affect the perception of competence to do the task and hence the motivation to do the task. Through the increase in comfort, attractiveness and satisfaction of lit environment, lighting can enhance the mood of the office workers making them happier and satisfied with their working environment. The main lighting parameters, lighting level, lighting distribution and light spectra, influence visual capability, comfort and perception, and thus, have to be optimized with respect to various office tasks to maximize benefits. In addition, complex social interactions take place at office on an everyday basis, such as meetings, presentations, training and marketing activities, involving personnel, customers, management teams and others. These can be controlled and orchestrated using the lighting infrastructure, as explained before. Wireless communication is very important as people are multitasking and on the move within the working place.

\section{Challenges}


In this section, we briefly discuss some challenges associated with the concept of iVLC. From the wireless communications standpoint, one important issue is how to implement the uplink. The lighting infrastructure inherently supports downlink transmission, but uplink requires some optical receivers to be installed on the ceiling. There is not necessarily need to have an array of receivers as large as that of the transmitters (i.e., LEDs). Uplink can use infrared communications, as typically assumed in VLC systems. One interesting solution that could be explored is to exploit the array of LEDs as an array of photodiodes. The LED junction is photosensitive and works as a photodiode, though it has not been optimized for such a function. During some intervals of time, some LEDs not being used as transmitter could be used as receivers. Even though their performance would be weak, having an array of them could provide acceptable results in the detection of uplink signals.

At system level, the complexity of the infrastructure could be considerable large. If the LED array is distributed across a large area, distributing data signals to all LEDs is not simple, particularly for high data rates. Programming the optical outputs could be also a difficult and time-demanding task, unless HLI programming languages are developed. Also controlling the whole system, e.g., interactive visual signals, data transmission and illumination, could be a complex and difficult task. From the point of view of usage, complexity should be hidden to users, and the system should have a highly intuitive user interface. Also, since spatio-temporal light patterns would be used to create visual interactions and signaling, it would be of fundamental importance to design a system whose light output is calm, pleasing, gently becoming an integral part of the ambient intelligence. Fluctuating light outputs could easily become a disturbing if the interactions are not designed properly. Cost-wise, this could be an interesting solution as the same infrastructure provides lighting, wireless access and visual interaction.

\section{CONCLUSIONS}

This paper introduced the concept of interactive visible light communications, where an array of RGB LEDs is used to provide lighting, wireless connectivity and means to create visual interaction and signalling. The iVLC idea was discussed in detail. Human light interaction was identified also as a novel area of development, focussed on the study of how light patterns in the space, time, color and intensity domains can be effectively used to on convey information to human beings. The paper discusses several promising environments where iVLC can be used. It is expected that optical wireless communications will be one of the enabling technologies of $6 \mathrm{G}$ [2]. Thus, VLC and iVLC are expected to have a very significant role in the future. In a more generic approach, iVLC can be seen as a particular case of the living surfaces concept [18], where mutiple functionalities (e.g., wireless communications, energy harvesting, signal processing, sensing, lighting) are implemented on surfaces using printed electronics technology.

\section{ACKNOWLEDGMENT}

The support from Academy of Finland in the projects HERONET and 6G Flagship Programme are greatly appreciated.

\section{REFERENCES}

[1] Vos T., den Breeijen P., van Drie W.D., "Quality and Reliability in SolidState Lighting: Qua Vadis?,” Solid State Lighting Reliability Part 2, Components to Systems, Edited by Willem Dirk van Driel. Jul. 2017.

[2] 6G white paper http://jultika.oulu.fi/Record/isbn978-952-62-2354-4

[3] Tsiatmas, A., Baggen, C., Willems, F., Linnartz, J.-P.M.G. and Bergmans, J. (2014). An Illumination Perspective on Visible Light Communications. Communications Magazine, IEEE. 52. 64-71. 10.1109/MCOM.2014.6852085.

[4] Li S., Pandharipande A., Willems F.M.J., Unidiractional visible light communiocation and illumination with LEDs, IEEE Sensors Journal, Volume: 16, Issue: 23 , Dec.1, 2016, pp. 8617 - 8626.

[5] Hammouda, M., Peissig, J. and Vegni, A.M., 2017, May. Design of a cognitive VLC network with illumination and handover requirements. In 2017 IEEE International Conference on Communications Workshops (ICC Workshops) (pp. 451-456). IEEE.

[6] Elgala, H., Mesleh, R. and Haas, H., 2010, September. Impact of LED nonlinearities on optical wireless OFDM systems. In 21st Annual IEEE International Symposium on Personal, Indoor and Mobile Radio Communications (pp. 634-638). IEEE.

[7] Teixeira, L., Loose, F., Brum, J.P., Barriquello, C.H., Reguera, V.A. and Dalla Costa, M.A., 2019. On the LED Illumination and Communication Design Space for Visible Light Communication. IEEE Transactions on Industry Applications, 55(3), pp.3264-3273.

[8] Popoola, W.O., 2016. Impact of VLC on light emission quality of white LEDs. Journal of Lightwave Technology, 34(10), pp.2526-2532.

[9] Gancarz, J., Elgala, H. and Little, T.D., 2013. Impact of lighting requirements on VLC systems. IEEE Communications Magazine, 51(12), pp.34-41.

[10] Figueiredo, M., Alves, L.N. and Ribeiro, C., 2017. Lighting the wireless world: The promise and challenges of visible light communication. IEEE Consumer Electronics Magazine, 6(4), pp.28-37.

[11] Kumar, N., 2013, March. Smart and intelligent energy efficient public illumination system with ubiquitous communication for smart city. In International Conference on Smart Structures and Systems, ICSSS'13 (pp. 152-157). IEEE.

[12] Ayub, S., Kariyawasam, S., Honary, M. and Honary, B., 2013, November. A practical approach of VLC architecture for smart city. In 2013 Loughborough Antennas \& Propagation Conference (LAPC) (pp. 106111). IEEE.

[13] Rea, M.S., \& Ouellette, M.J (1991). Relative visual performances: A basis for application. Lighting Research and Technology, 135-144.

[14] Wilbom, R.I., \& Carlsson, L. W. (1987). Work at video display terminals among office workers. In B. Knave \& P.G. Wideback (Eds.), Work with Video Display Units 86 (pp. 357-367). Amsterdam, the Netherlands: Elsevier Science.

[15] Flynn, J.E., Hendrick, C., Spencer, T., \& Martyniuk, O. (1979). A guide to methodology procedures for measuring subjective impressions in lighting. Journal of the illumination Engineering Society, 8, 95-110.

[16] Eklund, N.H., Boyce, P.R., and Simpson, S.N., (1999). Lighting and sustained performance. Illuminating Engineering Society of North America 1999 Annual Conference: Proceedings. IESNA: New York, NY. 501- 524.

[17] Laru, J., Katz M., Järvelä S. and Häkkinen J., Interactive Visible Light Communications: Using Human-Light Interaction in Learning Contexts, International Conference on Computer Supported Collaborative Learning, Gothenburg, Sweden, June 7-11, 2015.

[18] Katz, M. and O'Brien, D. (2019). Exploiting novel concepts for visible light communications: from light-based IoT to living surfaces. Optik. 195. 163176. 10.1016/j.ijleo.2019.163176. 